\section{Theragnostics: A Neologism That Generates Schism-The Hellenic Thesis}

TO THE EDITOR: Kyrie [sir]: Not as euphonic as theranostics, the term theragnostics, from the Hellenic therapevein [therapy] and "diagnosis," harmonizes well with "gnosis" and "prognosis" (1). The glossologic chasm that can be synthetized by the chresis [use] of the symbol "g" has been emphasized by a giant of Hellenic orthography and etymology, the academic Georgios Babiniotis: in theranostics, "nostics" refers more to the pathology than to diagnostics. Glossologically, the ideal is a synergy of the 2 words: "therapo-gnostics" (2).

In an epoch characterized by a plethora of polymorphous diagnostic and therapeutic methods, theragnostics becomes the base of modern clinical practice. However, the history of theragnostics - as the mastery of the synthesis of personalized biologic characteristics to generate the required diagnostic icons with the dynamics to practice analogous therapies - is not new. It had its genesis in the 1940s with radioiodine in diagnostic and therapeutic thyroidology. In November 1936, Karl Compton, president of the Massachusetts Institute of Technology, was asked to give a presentation at the Medical School of Harvard. His theme was, "What Physics Can Do for Biology and Medicine." Saul Hertz, who was there, asked whether it was technically possible to make iodine radioactive. The dialogue and the epistles between them set the base not only for the diagnosis and therapy of thyroid pathologies but also for the epoch of theragnostics (3).

Since then, radioactive iodine has been used for the scopes of pretherapeutic dosimetry; for the therapy of thyroid pathologies, including hyperthyroidism and carcinoma; and for posttherapeutic diagnosis and its characteristics, personalized. In this sphere, the parallel acme of a plethora of radiopharmaceuticals and diagnostic methods has generated a gigantic number of theragnostic radioisotopes and techniques. We will emphasize only some paradigms of the numerous radioisotope theragnostic practices.

Radiolabeled somatostatin receptor analogs such as ${ }^{111} \mathrm{In}-\mathrm{oc}-$ treotide, DOTATOC and DOTATATE labeled with ${ }^{177} \mathrm{Lu}$ or ${ }^{90} \mathrm{Y}$, and PET isotopes such as ${ }^{68}$ Ga-DOTATOC/DOTATATE are critical in neuroendocrine neoplasia (NEN) for staging the disease, planning its therapy, and monitoring the patient during and after therapy. Peptide receptor radionuclide therapy, the systemic therapy in advanced metastatic NEN, has altered oncologic clinical practice in gastroenteropancreatic NENs (2).

Radiopharmaceuticals that are synthesized by radiolabeling of prostate-specific membrane antigens (PSMA), such as ${ }^{68} \mathrm{Ga}-$
PSMA-HBED-CC, ${ }^{177} \mathrm{Lu} /{ }^{90}$ Y-J591, ${ }^{123} \mathrm{I}$-MIP-1072, ${ }^{131} \mathrm{I}$-MIP-1095, ${ }^{68} \mathrm{Ga} /{ }^{177} \mathrm{Lu}-\mathrm{PSMA}-\mathrm{I} \& \mathrm{~T}$, and DKFZ-PSMA-617, are basic to the personalized diagnosis and therapy of prostate carcinoma (4).

Our scope is not to analyze all the bibliographic and clinically practiced nuclear theragnostic techniques and radiopharmaceuticals. Our epithymia [longing] is to dynamitize the dialogue among epistemologists, academicians, physicians, and physicists in order to increasingly synchronize and harmonize our theragnostic techniques.

Instead of an epilogue, we would like to apologize for tyrannizing you with the Hellenic phraseology in our epistle and emphasize our eucharistia [gratitude] to the philoxenous [hospitable] $J N M$. The many paragraphs in this epistle were synthesized with the orthe chresis [correct use] of a plethora of Hellenic lexes. The first to practice the rhetoric of using a Hellenic lexicon was the academic Xenophon Zolotas back in 1957, who presented to the International Monetary Fund a lecture using only Greek words, with exeresis [exceptions] for articles and prepositions (5). We advise the readers to use a Hellenic lexicon as all English words in bold have a Hellenic origin.

\section{REFERENCES}

1. Frangos S, Buscombe JR. Why should we be concerned about a "g"? [letter]. Eur J Nucl Med Mol Imaging. 2019;46:519.

2. Verburg FA, Heinzel A, Hänscheid H, et al. Nothing new under the nuclear sun: towards 80 years of theranostics in nuclear medicine. Eur J Nucl Med Mol Imaging. 2014;41:199-201.

3. Fahey FH, Grant FD, Thrall JH. Saul Hertz, MD, and the birth of radionuclide therapy. EJNMMI Phys. 2017;4:15

4. Yordanova A, Eppard E, Kürpig S. Theranostics in nuclear medicine practice. Onco Targets Ther. 2017;10:4821-4828.

5. Zolotas X. Remarks by the Hon. Xenophon Zolotas, Governor of the Back of Greece and Governor of the Fund for Greece, at the closing joint session. Panhellenic School Network website. http://users.sch.gr/akouts/docs/Zolotas.pdf. Published September 26, 1957. Accessed March 30, 2021.

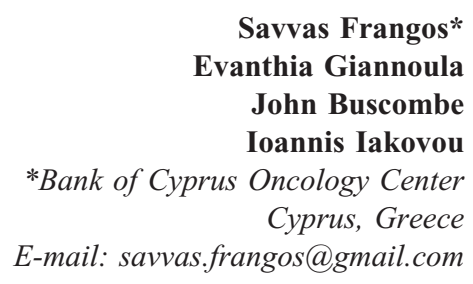

Published online Mar. 26, 2021. DOI: 10.2967/jnumed.121.262101 